\title{
LAS NUEVAS INFANCIAS, EL MISMO SUSTENTO. DESDE LA CONSTRUCCIÓN CURRICULAR DEL PROFESORADO EN EDUCACIÓN FÍSICA DE ARGENTINA
}

The new infancies, the same support. From the curricular construction of the faculty in physical education of Argentina.

Novas infâncias, o mesmo apoio. A partir da construção curricular de professores de educação física na Argentina.

\section{Gustavo Oscar Carnevale}

FaHCE - Universidad Nacional de La Plata. La Plata, Bs. As. Argentina. Instituto de Educación Superior “Miguel Neme”. Las Breñas, Chaco. Argentina. Telf.: 3731-516820. Correo electrónico: gustavocarnevalechaco@gmail.com

\section{Resumen}

Este trabajo analiza los discursos, sus relaciones y los sustentos epistemológicos que hacen posible algunas unidades discursivas, de las que tomamos el concepto de infancia, con cierto énfasis en la materialización del constructo "nuevas infancias", que se hace término (palabra) en el diseño curricular para la formación de profesores de Educación Física.

Encontramos en estas relaciones discursivas, dentro del currículo como práctica discursiva, que: infancia (nuevas infancias) responden a una red relacional compuesta por otros discursos, así nos permite la posibilidad de rastrear cuales son las condiciones de posibilidad que hacen que la infancia (nuevas infancias) develen su sustento epistémico bajo el posicionamiento que subyace a los conceptos de cuerpo, aprendizaje, enseñanza y sujeto.

Para la tarea de problematizar tomamos como corpus teórico las investigaciones y posicionamientos de la educación corporal propuesta por Crisorio, Giles y sus equipos, con el fin de avanzar en deconstruir aquello que se dice, intentando llegar a qué condiciones epistemológicas permiten que así se diga, a la vez que se propone en contraposición la 
posibilidad de poder pensar otras opciones que solo puede ser posible bajo el corrimiento teórico, de sustento y significados de los mismos términos pensados desde otros puntos de vista.

Palabras clave: nuevas infancias, diseño curricular, educación corporal.

\begin{abstract}
This paper analyzes the discourses, their relationships and the epistemological underpinnings that make possible some discursive units, from which we take the concept of childhood, with a certain emphasis on the materialization of the construct "new childhoods", which becomes a term (word) in the curricular design for the formation of Physical Education teachers.
\end{abstract}

We find in these discursive relationships, within the curriculum as a discursive practice, that: childhood (new childhoods) respond to a relational network composed of other discourses, thus allowing us the possibility of tracking which are the conditions of possibility that make childhood (new infancias) reveal their epistemic sustenance under the positioning that underlies the concepts of body, learning, teaching and subject.

For the task of problematizing we take as a theoretical corpus the investigations and positioning of corporal education proposed by Crisorio, Giles and his teams, in order to advance in deconstructing what is said, trying to arrive at what epistemological conditions allow that to be said, At the same time, it proposes, in contrast, the possibility of being able to think of other options that can only be possible under the theoretical shift, of sustenance and meanings of the same terms thought from other points of view.

Keywords: new childhoods, curricular design, body education.

\title{
Resumo
}

Este artigo analisa os discursos, suas relações e os fundamentos epistemológicos que possibilitam algumas unidades discursivas, das quais tomamos o conceito de infância, com uma certa ênfase na materialização da construção de "novas infâncias", que se torna um termo (palavra) na desenho curricular para a formação de professores de Educação Física. 
Encontramos nessas relações discursivas, dentro do currículo como prática discursiva, que: a infância (novas infâncias) responde a uma rede relacional composta por outros discursos, permitindo-nos a possibilidade de rastrear quais são as condições de possibilidade que fazem a infância (nova infantes) revelam seu sustento epistêmico sob o posicionamento que está subjacente aos conceitos de corpo, aprendizagem, ensino e sujeito.

Para a tarefa de problematizar, tomamos como corpus teórico a pesquisa e o posicionamento da educação corporal proposta por Crisorio, Giles e suas equipes, para avançar na desconstrução do que é dito, tentando chegar ao que as condições epistemológicas permitem que isso seja dito, Ao mesmo tempo, propõe, em contraste, a possibilidade de pensar em outras opções que só podem ser possíveis sob a mudança teórica, de sustento e significados dos mesmos termos pensados a partir de outros pontos de vista.

\section{Palavras-chave: novas infâncias - desenho curricular - educação corporal.}

\section{La construcción curricular como encuadre}

Este trabajo tiene como fuente documental las Recomendaciones para la elaboración de diseños curriculares para los Profesorados de Educación Física (INFOD, 2009) y los Diseños curriculares jurisdiccionales de doce provincias argentinas, que se desprenden del primer documento mencionado ${ }^{1}$. Se analiza en dicha documental los discursos que articulan determinados conceptos y las configuraciones que se construyen entre ellos.

Por ello, la práctica de investigación rastrea el proceso mediante el cual un conjunto de enunciados hace posible, de acuerdo con la relación que guardan entre sí, la formación de unidades de discurso (Emiliozzi, 2017).

Se construye metodológicamente, apoyado en lo que Emiliozzi denomina "El método del discurso y sus relaciones epistemológicas" (Emiliozzi, 2017). Por tanto las exiguas referencias

\footnotetext{
${ }^{1}$ Dado que Se observó una linealidad conceptual entre los posicionamientos teóricos sugeridos por el INFOD y la concreción de los instrumentos jurisdiccionales se decidió citar sólo el documento del INFOD, muchas de estas citas y posiciones se encuentran transcriptas en los diseños jurisdiccionales, y sus ideas son replicadas con carácter axiomático, salvo muy escasas excepciones.
} 
sobre la construcción curricular, sus formas y sus lógicas, no son más que un mínimo encuadre de la materialización de los discursos analizados. Ya que no se analizan los documentos y su construcción como objeto de estudio, no se toma el autor, las formas, las posiciones, como objeto de estudio, sino como aquello que ha sido materializado y de lo que podemos servirnos para el análisis de las formaciones discursivas operantes.

Al comenzar un análisis del discurso, ya sea de leyes, documentos, normas, etc., se nos presentan los autores que permitieron determinado tipo de documento; pero en el método que pretendemos poner en práctica, no se procura establecer las ideas que éstos tienen. Un sujeto, a diferencia de un ciudadano o una persona, esta precedido por el discurso, "lo que implica, necesariamente, que primero haya actuado la articulación de los significantes" (Eidelsztein, 2012: 18). En efecto, ante el enunciado de un discurso, lo que se trata de analizar son las condiciones de producción, no las representaciones e ideales del autor (Emiliozzi, 2017).

Desde allí surge como categoría: el concepto de infancia, que se presenta atravesado y configurado por los enunciados que sustentan los conceptos de: cuerpo, aprendizaje, enseñanza y sujeto, a partir de los que se develan los sustentos epistemológicos que lo hacen posible desde el currículo como instrumento teórico-técnico-administrativo que configura la formación de profesores de Educación Física, en los Institutos de Educación Superior no universitarios².

Considerando que la construcción curricular de la formación, no puede ser otra cosa que un reflejo de los saberes, supuestos, posicionamientos, significados y sustentos epistemológicos circulantes en el campo, al mismo tiempo que se considera a la formación como un dispositivo eficaz para asegurar su reproducción.

El currículum, su implementación, ha condicionado nuestras prácticas. Es un componente instituyente, pues, de la realidad educativa que vivimos; podría decirse que la conforma. Si bien,

\footnotetext{
${ }^{2}$ La formación de profesores de Educación Física en Argentina, según la Ley de Educación Nacional 26.206/06, se encuentra a cargo del nivel superior en la estructura del sistema educativo que dicha Ley regula, dentro del cual se albergan los Institutos de Educación Superior no Universitarios y Las Universidades.
} 
las prácticas dominantes en un momento dado también lo condicionan a él; es decir, que el currículum es a la vez instituido en su realización (Gimeno Sacristán, 2010:12).

Si consideramos al currículo como una práctica discursiva nos revela un discurso, un orden del discurso para decir con Foucault (1992) que determina, tanto la inclusión, como exclusión de ideas, conceptos y saberes. Siguiendo a Foucault, se interpreta cuales son las condiciones de posibilidad que sólo pueden permitir, y a su vez remitir, a determinados discursos-enunciados, que inmediatamente excluyen a todos los demás, "el discurso no es simplemente aquello que traduce las luchas o los sistemas de dominación, sino aquello por lo que, y por medio de lo cual se lucha, aquel poder del que quiere uno adueñarse" (Foucault, 1992:6).

Se entiende entonces que las formaciones discursivas que develamos en el análisis de nuestras fuentes van a determinar con una fuerza tan sutil como gravitacional, no sólo la formación, sino también la Educación Física como campo disciplinar, y por consiguiente los conceptos que en ella operarán en forma de bucle, ya que han sido estos discursos lo que han permitido la misma construcción curricular.

Hay algo que hace que un determinado texto forme parte de una disciplina y no basta que este esté bien formulado en una lengua. Para producir un texto de una disciplina es necesaria una reducción. Esa reducción es denominada formación discursiva, la cual no opera como estructura en el sentido estructuralista, sino que es una reducción que tiene condiciones que son absolutamente históricas, institucionales, sociales, no son condiciones formales (Emiliozzi, 2017).

Se fundamenta esta afirmación, tanto desde las teorías curriculares como desde el análisis de la constitución del campo de la Educación Física y sus prácticas en Argentina

...la Educación Física, en tanto disciplina tal como se la reconoce en la actualidad, no nace como muchos autores han indicado estrictamente con la Ley 1.420 “de Educación Común" de 1884 que da origen al Sistema Educativo argentino, sino que es resultado de un doble proceso contiguo conformado por la fundación y fundamentación de su 
práctica y por la institucionalización de su oficio, como modos de reproducción de sentidos disciplinares relativamente homogéneos y autónomos (Galak, 2014).

Dentro de un aparentemente ecléctico corpus teórico, se identifican enunciados que pueden pasar inadvertidos bajo una "renovada" manera de formalizarse, pero que mantienen un rasgo epistémico común, y que no es otro que el de la Educación Física misma, en este caso, circunstancialmente analizados dentro de la formación inicial.

Los pensamientos y los conceptos que se acuñaron para la construcción política logran, muchas veces, su cometido. Es entonces cuando son aceptados y reconocidos por las grandes mayorías y se vuelven sentido común, o bien son sancionados jurídicamente y se integran al sistema normativo (INFOD, 2009:7).

El currículo toma como fundamentos epistémicos los principios de paradigmas como: el socio-crítico, la teoría de la complejidad de Edgar Morín, derivada de la teoría general de los sistemas $^{3}$, es así que "la influencia de la Escuela de Frankfurt, con su modelo socio-crítico, las concepciones holísticas y el paradigma de la complejidad, entre otras, contribuyen a sustentar un nuevo enfoque disciplinar"(INFOD, 2009:19) desde donde se desprenden términos técnicos y conceptos que encuadran epistemológicamente a la formación en Educación Física, conformando una red de significados cuya influencia se transponen a la Educación Física como campo.

En líneas generales esta red de significados, funcionando como dispositivo discursivo con asiento en la corporeidad, la motricidad humana: "La emergencia de este nuevo paradigma de pensamiento posibilita advertir las diferentes dimensiones y aspectos relevantes de la corporeidad y motricidad humanas" (INFOD, 2009:15) pero también sustentada en la complejidad del ser humano, la contextualización de los contenidos, la centralidad del sujeto que aprende, la naturaleza humana, entre otros términos técnicos, dan origen a lo que interesa y se problematiza: las formaciones discursivas que

\footnotetext{
3 Se considera necesario como herramienta problematizadora, hacer referencia a la tesis sobre aprendizaje motor de Rocha-Bidegain que plantea: "La teoría general de sistemas define como sistema a un complejo de elementos interactuantes y pretende establecer principios generales para todos los sistemas, independientemente de su naturaleza física, biológica o sociológica. La comprensión de la realidad será a partir de su totalidad, y los niveles de dependencia entre los elementos variarán en función del tipo de sistema al que se haga referencia, volviendo así a resaltar la importancia del todo y de las redes de relaciones (Rocha-Bidegain, 2012, p.60-61).
} 
hacen emerger a la infancia, en tanto atravesada por los discursos que construyen las concepciones de: Aprendizaje, sujeto, cuerpo y enseñanza.

\section{Las nuevas infancias ¿una novedad?}

Tras el encuadre que justifica la elección de las fuentes analizadas, se toma como herramienta para repensar el lugar, la significación y el sentido de la/s infancia/s (y los elementos que la configuran: cuerpo, aprendizaje, enseñanza y sujeto) a la construcción teórica que la Educación Corporal, como programa de investigación científica en curso. Y se focaliza la atención en la selección de contenidos que se ha realizado para dar forma al currículo de la formación inicial en Educación Física, reconociendo los criterios propuestos para ello, como así también los fundamentos que subyacen en dicha selección, pensándolos a estos en tanto formaciones discursivas que hacen posible la emergencia de conceptos.

Y como se adelantó, se pone en el tapete de la problematización el concepto de infancia, determinando como se arma un "haz de relaciones" (Emiliozzi, 2017) cada vez que se enuncia cuál es la enseñanza que se propone para esa infancia, cuál es el cuerpo que se encuentra implicado desde estas posiciones y cuál el concepto de aprendizaje, lo que necesariamente da cuenta de una concepción de sujeto.

No es posible analizar de manera estanca ninguno de los cuatro conceptos (cuerpo, aprendizaje, enseñanza y sujeto) no sólo por su inviabilidad tratándose del currículo que conducirá la formación de profesores de Educación Física, sino más bien por que de hacerlo se incurriría en un error epistemológico y metodológico. Es necesario, teórica y metodológicamente el análisis de estos conceptos como elementos covariantes ${ }^{4}$, en la conformación del discurso operante que da origen a determinada concepción de infancia. Alguna redes relacionales de significación muestran que uno de los conceptos mas fuertes es "el aprendizaje", es el concepto que actúa como guía en la enseñanza "Toda acción de enseñanza se basa explicita o implícitamente en una concepción del aprendizaje (Renzi y Ferrari, 2008. Cit. En INFOD 
2009:58) en un amarre indisoluble a una concepción determinada de sujeto. Los contenidos a enseñar, y en relación con su forma de enseñarlos, se seleccionarán en referencia al aprendizaje y a las teorías que lo guían, conformando así un criterio que se declara de manera tanto explicita como implícita.

Toda selección que se realice puede apoyarse en diversos criterios y responder a múltiples razones pero, indudablemente, está condicionada por los propósitos expresos del proceso formativo. [...] esta selección implica un debate epistemológico, político e ideológico sobre el conocimiento en sentido histórico, [...] Es decir, debe asegurar los modos de pensamiento fundamentales en el campo o disciplina de que se trate, debe ser capaz de justificar la exigibilidad de los contenidos que plantea, o sea, debe responder sobre: ¿qué tipo de argumentos avalan la inclusión?, ¿por qué es necesario exigirle a los estudiantes su dominio? Y, además, deben asegurar su transmisibilidad (INFOD, 2009:31).

La selección de contenidos para la enseñanza, con base en el aprendizaje ubicando al sujeto como centralidad

... se orientan a la búsqueda del equilibrio y la armonía internas [del documento como práctica discursiva], con clara referencia a fuentes filosóficas y psicológicas que amplían la consideración biologicista reducida del cuerpo [aunque nada se diga del cuerpo natural] y tienen en cuenta, además, los contextos socioculturales en que los sujetos viven y su complejidad emocional, relacional, cognitiva y ética” (INFOD, 2009:18).

Estas sugerencias habilitan y dan lugar a la voz de la teoría de la complejidad, en la que se sustentan los diseños curriculares para definir discursivamente: la/las infancias, cada vez que se develan los discursos que actúan como telón de fondo en los conceptos de enseñanza, aprendizaje, cuerpo y sujeto. La teoría de la complejidad es propuesta por Morin, como el reordenamiento de una "nueva physis" con la lógica de un sistema (natural en intercambio con lo

\footnotetext{
4 "grupo de elementos que forman un conjunto covariante que implica cierto número de coordenadas" (Lacan, 1956: 160). Elementos que no pueden ser interpretados por separados, y que la variación, o dirección tomada por alguno de estos elementos implica necesariamente la variación y direccionamiento de los demás.
} 
social y cultural) siempre y cuando haya un sustento esencialmente humano (natural e incluso orgánico/biológico) sobre el cual asentarse:

El hombre sólo se completa como ser plenamente humano por y en la cultura. No hay cultura sin cerebro humano (aparato biológico dotado de habilidades para actuar, percibir, saber, aprender), y no hay mente (mind), es decir capacidad de conciencia y pensamiento sin cultura" (Morin, 1999:24).

Es con este rumbo, y bajo el paraguas de la complejidad moriniana que aparecen contenidos formalizados en constructos como: nuevas infancias; caracterización de niñez y la adolescencia como fenómeno histórico, social y cultural; culturas infantiles y juveniles; constitución de la subjetividad en escenarios de pobreza y exclusión; entre otros. Dejando ver ahí una concepción del "sujeto" montado sobre la idea de "individuo". Sujeto al que se psicologiza en la medida que se le otorga un interior capaz de percibir, sentir, aprender, emitir palabras y relaciones críticas, a través de un sistema nervioso central (cerebro), que tendría capacidad de adaptación u organización acorde a su etapa evolutiva y el contexto "cultural", según el intercambio que se produzca. Se puede fundamentar, desde la simple deducción lógica que representa la organización de los contenidos seleccionados, ya que dichos contenidos se derivan del campo de la formación específica, que incluye la unidad curricular Psicología, algunos de sus contenidos son: "las fases etarias y sus perfiles psicológicos; niñez, adolescencia, juventud, adultez y vejez; el desarrollo psico y sociomotor; relación entre los aspectos psico y biogenéticos, sociales y culturales en el desarrollo de los sujetos; teorías del aprendizaje; el aprendizaje motor" (INFOD, 2009:44). Pero por elección metodológica, son los discursos que allí funcionan y se fusionan los que problematizamos.

Este proceso de psicologización, con fuerte incidencia en la caracterización de la/s infancia/s, también se presenta en aquellas unidades que refieren explícitamente a los saberes puramente bio-fisiológicos, con el agregado de saberes de la neurología y las neurociencias, encontrándose contenidos como estos en la unidad curricular Biología humana:

fisiología general y funcionalidad de los diferentes sistemas orgánicos en relación con la 
actividad motriz en las diferentes edades; neurofisiología en la fundamentación de las acciones motrices; biomotricidad; análisis de acciones motrices; componentes anatómicos, biomecánicos, fisiológicos y neuromotrices; su relación con los aspectos cognitivos, emocionales y sociales del sujeto (INFOD, 2009:44).

Sin perjuicio de esta simple, pero concisa argumentación, en base a la organización, que da cuenta de lo que Gimeno Sacristán asevera:

El currículum desempeña una doble función —organizadora a la vez que unificadorade la enseñanza y del aprendizaje, por un lado, a la vez que, por otro lado, se produce la paradoja de que en él se refuerzan las fronteras (y murallas) que delimitan sus componentes, como por ejemplo la separación entre las asignaturas (asignaciones) o disciplinas que forman su contenido (Gimeno Sacristán, 2010:22).

Y escudriñando más profundamente, se puede afirmar que las "nuevas infancias" (como término novedoso) a las que hace referencia la construcción curricular, no es más que un concepto "adornado" del individuo como "ser integral": bio-psico-social, al que bajo las teorías de la complejidad, se pone en un contexto/medio del que devendrá una taxonomización, siempre pensando en las características psicológicas y su relación con el medio, como discurso que justifica la singularidad de los sujetos, deja de pensarse en "el niño" como genérico y universal, y se pasa a pensar en "las infancias" (también "las adolescencias", "las culturas juveniles" etc).

Esto significa incorporar algunas de las problemáticas vigentes, a saber: las nuevas configuraciones familiares, las nuevas infancias y adolescencias, las diferentes formas de relación con el Estado, los diversos modos de organización social, el lugar que ocupa el trabajo en la sociedad actual, entre otros (INFOD, 2009:33).

Sigue operando el mismo concepto de sujeto como individuo, ahora como miembro de un subgupo dentro de la especie humana: el sujeto (individuo) del aprendizaje en la/s infancia/s, que en relación con el medio y el contexto, clasificará aunque lo niegue, por ejemplo en infancias pobres, infancias ricas, infancias felices, infancias sufridas, infancias tecnológicamente influenciadas, etc. 
Si bien es preciso reconocer que "las infancias" pensada ahora, con el trasfondo de la idea de grupo, o la de subgrupo tiene una ascendencia social y una descendencia sociológica, mantiene la negación sistemática a dejar de lado la constitución bilógica, orgánica, esencial de una naturaleza que da asiento u origen a lo cultural, social e histórico. Lo que representa una fidelidad incondicional a las mismas bases epistemológicas que sustentan el cuerpo, el aprendizaje, la enseñanza y el sujeto. Tal como dicen Giles y Rocha-Bidegain: "En síntesis todo el campo del aprendizaje motor se ha organizado a partir de la idea que existe una naturaleza humana que orienta y determina los comportamientos y posibilidades de aprendizaje" (Giles y Rocha-Bidegain, 2015:85).

Es así que ahora, las nuevas infancias seguirán siendo un estadío biológico-psicológico-natural, complementado por lo contextual e histórico en una nueva compilación taxonómica, que no es suficiente para representar a quienes son sujetos de las prácticas que habitan.

Como vemos se producen relaciones complejas pero a su vez consistentes entre los discursos que arman el concepto de infancia, bajo los enunciados que determinan: un cuerpo natural, un sujeto (social) que es determinado desde la psicología como individuo, al que se pretende enseñar, regido por sus posibilidades de aprender.

\section{Una posible opción}

En esta parte del trabajo, se intenta un ensayo que propone básicamente intentar nuevas relaciones discursivas. Sin agotar todas las posibilidades que podrían emerger una vez que se desarticula el discurso presente y su poder de dispositivo, que funciona a modo de constructor de infancia/s, mediada por los conceptos de cuerpo, aprendizaje, enseñanza y sujeto. Posibilidad de subversión que sólo puede tener asiento en una nueva fuente epistemológica, que inaugure otro punto de vista a modo de ruptura y no de continuidad "Si el discurso funda el objeto del cual va a hablar, si el objeto de estudio es producido por los enunciados, un discurso se funda en la separación que establece respecto de otro, al que obviamente excluye" (Crisorio 2015:8). 
Retomando el posicionamiento teórico de la Educación Corporal sobre los conceptos a los que se considera como parte del núcleo duro de dicha teoría: la concepción de sujeto, cuerpo y práctica corporal, desconectándolos del concepto de aprendizaje y estableciendo nuevas maneras de pensar la enseñanza, se arman nuevas relaciones discursivas que habilitan un corrimiento respecto del concepto de infancia que presentamos como operante en el discurso curricular.

En primer lugar la teoría de sujeto, que se entrelaza necesariamente a una determinada concepción de cuerpo "Ninguna educación puede, entonces, pensar ni presentar el cuerpo sin hacer referencia a ese otro elemento al que, transformado en el campo de la filosofía y de la ciencia, hoy se llama sujeto" (Crisorio, 2015:10). Y en segundo lugar, y dentro de la perspectiva teórica que utilizamos como herramienta para problematizar se toma la definición de prácticas corporales (como contenidos a enseñar), como otro de los elementos necesarios para sustentar las rupturas de ciertos significados. Crisorio define las prácticas corporales como un concepto novedoso:

A su vez, cuando se acuñan conceptos nuevos no es como sinónimos de otros existentes: prácticas corporales, por ejemplo, no es un equivalente de actividades físicas o de movimiento humano, sino que indica las prácticas históricas, por ende políticas, que toman por objeto al cuerpo (Crisorio, 2015:9).

Problematizar las construcciones discursivas de los currículos y triangular con estos conceptos novedosamente presentados permitiría dejar de pensar en sujetos como individuos de una especie (con una naturaleza intrínseca) agrupados en un subgrupo que se conforma en relación con el medio/contexto del que forman parte, que pueden ser caracterizados, aunque bastante indefinidamente, pero homogeneizados en sus diferencias, incluso en la pluralización semántica a la que se apela por medio del término infancias. El sujeto que propone la educación corporal implica abandonar al sujeto emergente de los discursos curriculares con un anclaje firmemente constituido en su naturaleza humana (ergo en su otologización como: "human") representada en el cuerpo de quienes constituyen las infancias, complementada por el anexado socio-contextual-emocional-sensible tal como lo define la corporeidad: 
El ser humano es cuerpo biológico, pero al mismo tiempo cuerpo sensible, inteligente, social. Hablar del cuerpo en toda su amplitud es trascender el sistema orgánico, para entender y comprender al propio "humanes". El ser se constituye con un cuerpo, pero no es un cuerpo exclusivamente objetual, es un cuerpo que vive, que es acción y expresión (INFOD, 2009:19).

Implica también desmantelar la idea de: necesidad de conocer las Infancias (como conjunto de individuos-sujetos con posibilidades de aprendizaje) con el fin de adecuar los contenidos (las prácticas corporales) para su enseñanza, con el objetivo de que esta adecuación permita el acceso a ellas.

“La Educación Corporal piensa al sujeto como producto de una articulación significante, como una novedad, por completo distinta del individuo, la persona, el hombre" (Crisorio, 2015:10). Es decir, es necesario dejar de pensar en sujetos (en plural) para pensar en el sujeto (como término singular que no admite la pluralización) y pensar la enseñanza haciendo foco en el saber como aquello necesario de ser transmitido en una cultura, con el objetivo de permitir el acceso o el mejor aprovechamiento de ella, considerándolo como saber universal que no se adecuará al contexto, ni modificará sus lógicas pensando en quienes aprenden (sea cual sea la taxonomizacion de las infancias), teniendo como norte que es ese saber el que habilita el quiebre de la reproducción, y permite un acercamiento a la "igualdad" en términos de justicia de distribución cultural, bajo la intención política de universalizar su transmisión, sin recetas para la enseñanza.

Constituyéndose de esta manera un sujeto particular, que no puede escapar de la universalización con la sola incorporación del concepto de infancias. Un sujeto que es efímero, que se construye en el lenguaje y que no se puede materializar, tipificar, ni cristalizar, ya que no será el mismo la próxima vez que se encuentre en una situación de enseñanza, aunque se intente forzosamente inmovilizarlo dentro de los límites de las nuevas infancias. Así entonces, será el saber que los media y la red de significantes quienes aportarán puntualmente a su construcción y no el contexto/medio que comparten. 
Pensar el cuerpo humano y el sujeto constituidos en el orden simbólico y no en la naturaleza, pensarlos como lo que resulta de la desconexión en el hombre de lo humano y lo animal, y no de su articulación o conjunción, lleva a pensar también de otro modo el problema de la educación, tanto en sus puntos centrales como en sus detalles (Crisorio, 2015:10).

Se propone a su vez, pensar que no se enseñarán las prácticas corporales (juegos, deportes, gimnasia, danza, prácticas corporales en el medio natural, etc.) según se configuren "las nuevas infancias” sino según las lógicas propias de las prácticas, esto implicará entonces, enseñarlas sin pensar en las características de "la/s infancia/s" como guía que reduce a la categoría de "medios" a las prácticas corporales en la Educación Física, según la propuesta curricular y los discursos que dan origen a su materialización.

A partir de nuevas configuraciones discursivas que estas posiciones y teorías arman, permite dejar de pensar en un "deporte social" (deporte para las infancias en situaciones de pobreza o vulnerabilidad social), “deporte escolar” (en el ámbito escolar, con adecuaciones tendientes a evitar la competitividad en base a prescripciones psicológicas que caracterizan a las diferentes infancias), "juegos en contexto intercultural" (intentando forzar formas de juegos en juegos con lógicas propias), "juego para la infancia o la adolescencia” (determinando de manera a-priori y sesgada los intereses que podrían operar) , "gimnasia en infancias carentes" (transponiendo intencionalidades), "gimnasia para oficinistas" (sobrevalorando lo instrumental en perjuicio del saber), "gimnasia en situaciones de encierro" (pensando en una subjetivación estandarizada) etc.

En las relaciones de sujeto, enseñanza, cuerpo, prácticas corporales (contenidos como mediador entre enseñante y aprendiz, en lugar del aprendizaje como guía) que se tejen bajo estos discursos puede pensarse entonces en la posibilidad de concebir las prácticas corporales como esas prácticas que por sus lógicas internas constitutivas practican a quien las practica, en la edad cronológica, caracterización social o relación contextual que sea. Como prácticas corporales que hacen cuerpos, que sólo suponen un sujeto, que se enseñan en relación a la propia práctica y sus lógicas y que no requieren saber nada del aprendizaje. 


\section{Más que un cierre...una invitación}

Se puede decir que: si el sujeto es realmente pensado como una construcción socio-histórica del lenguaje, como afirma Crisorio, que no puede ser materializado ni clasificado; que son las prácticas corporales las que practican y toman por objeto al cuerpo ya que existen antes del sujeto, haciendo de él una construcción que no tiene asiento ni esencia en lo biológico, ni en nada natural; que habría que abdicar de la falsa idea de democratización pensada como la adecuación contextual del contenido a las infancias, para pensar en la democratización en el sentido de universalizar el contenido en su enseñanza; que el aprendizaje tomado como guía para la enseñanza asentado en las teorías del aprendizaje, sólo puede tener existencia en la medida que se sostenga la naturaleza humana.

No se puede mas que proponer: prescindir definitivamente de la categoría de infancia/s (o nuevas infancias) como organizador de la enseñanza, o como elemento referencial en la Educación Física, esto exime también de la preocupación por el aprendizaje como criterio de selección de contenidos destinados a esas infancias.

En la medida en que la/s infancia/s, son declaradas como una construcción sociohistórica-cultural, es necesario lógicamente desvincularla de un "ser", de un "deber ser", de toda pretensión natural, de la enseñanza y a su vez, esta del aprendizaje. Ya que la/s infancia/s sólo podrían "ser", como postula la Educación Física, en la medida que exista algo natural como piedra fundacional a lo que se "complementa" ad hoc para que cierre en una nueva propuesta como son las "nuevas infancias". Es justo reconocer que estas relaciones abren la puerta a un variado numero de problemas e implicancias dentro de un sistema que esta pensado en base a "la naturaleza humana", a un individuo (nombrado como sujeto) con capacidades, potencialidades, condicionantes, limitaciones de aprender, de orden innato (natural - bilógico). Al que se puede materializar, con mayor o menor grado de incertidumbre, con mayor o menor influencia socio-cultural, pero materializar al fin. Estos problemas pueden dar origen a ciertas preguntas cómo: ¿Cómo se organiza la escolarización, la formación y la Educación Física sin el concepto de infancia/s? ¿Se podría organizar la Educación Física si no existiese: la/s infancia/s, la adolescencia, la adultez y la vejez como conceptos gravitantes en la enseñanza? ¿Qué sería de la 
Educación Física si se centrara en el saber de las prácticas corporales como recorte cultural a ser transmitido sin pensar en el aprendizaje? ¿Qué consecuencias tendría sobre los planteamientos didácticos de la Educación del cuerpo, la prescindencia del concepto de infancia/s?

Quizás declarar que no se cuenta con respuestas cerradas a estas preguntas pueda hacer considerar que lo expuesto no es más que una crítica idealista sin sentido; una posición ideológica utópica; una propuesta desconectada del funcionamiento socio-institucional; una manera de decir lo mismo en diferentes términos.

O quizás pueda movilizar a la problematización en búsqueda de más preguntas, considerando lo propuesto como una apuesta política; puede que quizás pueda ser entendida como el verdadero sentido político que se le reclama a la educación; o como la democratización de la educación en el sentido de ruptura del orden establecido, lo que representa el verdadero valor de lo democrático.

Que pueda pensarse cualquiera de estas cosas, como así cualquier otra, sobre lo propuesto, no es más que otra muestra contundente de que, entre el que expone, el que lee y el asunto que se trata, se construye un sujeto puntual, particular, efímero, incierto e inasible.

\section{Referencias bibliográficas}

Carballo, C. (Coord.) (2015). Diccionario Crítico de la Educación Física académica. CABA: Prometeo.

Crisorio, R. (coord.) (2015). Ideas para pensar la educación del cuerpo La Plata: Edulp (REUN).

Emiliozzi, M. (2017). El método del discurso y sus relaciones epistemológicas. apuntes desde la obra de Michael Foucault. Revista de Ciencias Sociales (Cl) [en línea], [Fecha de consulta: 2 de diciembre de 2017] Disponible en:〈http://www.redalyc.org/articulo.oa?id=70852271001>ISSN 0717-2257

Foucault, M. (1992). El orden del discurso. Buenos Aires: Tusquets Editores. 
Galak, E. (2014). Qualquer semelhança com a realidade (não) é mera coincidência. La constitución de las bases epistémicas de la educación física (argentina) frente a la construcción actual de la disciplina. Revista Brasileira Ciências do Esporte, Florianópolis, 36, 2, 253-267.

Giles, M- Rocha-Bidegain, L. (2015). Aprendizaje motor "en” Carballo, C. (Coord.). Diccionario Crítico de la Educación Física académica. (pp. 79-86)

Gimeno Sacristán, J. (comp.) (2010) Saberes e incertidumbres sobre el curriculum. Madrid: Morata.

Ministerio de Educación, INFOD. (2009). Recomendaciones para la elaboración de diseños curriculares, Profesorado de Educación Física. $1^{\circ}$ edición, Buenos Aires.

Morin, E. (2009) Introducción al pensamiento complejo. Gedisa (1994). Los 7 saberes necesarios para la educación del futuro. Paris: UNESCO.

Rocha- Bidegain, L. (2012). "El aprendizaje motor: una investigación desde las prácticas”. (Tesis de Maestría, Maestría en Educación Corporal) FaHCE-UNLP La Plata. Recuperado de www.fahce.unlp.edu.ar/memoriaacademica 